\title{
Maximizing the Net Present Value of a Steiner tree
}

\author{
K.G. Sirinanda - M. Brazil • \\ P.A. Grossman • J.H. Rubinstein • \\ D.A. Thomas
}

Received: date / Accepted: date

\begin{abstract}
The theory of Steiner trees has been extensively applied in physical network design problems to locate a Steiner point that minimizes the total length of a tree. However, maximizing the total generated cash flows of a tree has not been investigated. Such a tree has costs associated with its edges and values associated with nodes. In order to reach the nodes in the tree, the edges need to be constructed. The edges are constructed in a particular order and the costs of constructing the edges and the values at the nodes are discounted over time. These discounted costs and values generate cash flows. In this paper, we study the problem of optimally locating a single Steiner point so as to maximize the sum of all the discounted cash flows, known as the Net Present Value (NPV).

An application of this problem occurs in underground mining where, we want to optimally locate a junction point in the underground access network to maximize the NPV. We propose an efficient iterative algorithm to optimally locate a single degree-3 Steiner point. We show this algorithm converges quickly and the Steiner point is unique subject to realistic design parameters.
\end{abstract}

Keywords Network optimization - Optimal mine design · Net Present Value . Steiner points.

K.G. Sirinanda $(\bowtie)$ · P.A. Grossman · D.A. Thomas

Department of Mechanical Engineering, The University of Melbourne, Melbourne, Victoria 3010, Australia.

Tel.: +61383446734

Fax: +6138344 4290

E-mail: kash.s@student.unimelb.edu.au

M. Brazil

Department of Electrical and Electronic Engineering, The University of Melbourne, Melbourne, Victoria 3010, Australia.

Tel.: +61383446699

Fax: +61383447412

J.H. Rubinstein

Department of Mathematics and Statistics, The University of Melbourne, Melbourne, Victoria 3010, Australia.

Tel.: +61383445550

Fax: +6138344 4599 


\section{Introduction}

The Steiner tree problem deals with constructing a minimum cost tree to interconnect a given set of locations known as terminals. In practical applications the associated costs may vary with time, making this optimization problem particularly hard to solve. Such trees may have construction and operational costs associated with their edges and values associated with their terminals. Further, the costs and values which generate cash flows in the trees may be discounted over time. Therefore time has a crucial impact on the value of such trees. The discount rate is the key variable of this process and is used to discount the cash flows. The sum of all the cash flows is known as the Net Present Value (NPV).

This paper is motivated by a problem in optimal mine design which is to locate a junction point in the access network of an underground mine to maximize the $\mathrm{NPV}$. If the location of the junction point is known, the process of constructing the mine access can be scheduled. To date, the scheduling and access design of an underground mine have only been considered as two separate optimization problems. A weakness of this approach is that the costs of access construction fail to be reflected in the NPV calculation. In this paper we formulate scheduling and access design as a single optimization problem.

In the literature, the problem of underground access design is generally treated as a tree network problem where, the locations of the ore resource draw points are given and the junction points of the network are to be obtained for a given objective function. To find a minimum length network connecting a set of given terminals or points, Steiner points or junctions can be added to reduce the network length. This is the classical Steiner problem [1] and in the Euclidean minimal Steiner tree, the angles at the Steiner point are exactly $2 \pi / 3$. However, the problem we need to analyse is different from this, as the Steiner points will be located to maximize the NPV. The optimization of underground mine access has been studied by Brazil et al $[2,3]$ to minimize the development and haulage costs over the life of a mine. They modelled the problem as a variation of the Steiner problem with a constraint on the gradient in order to ensure navigability by trucks. The gradient constraint defines a safe climbing limit for trucks, typically in the range 1:9 to 1:7. However, the discounted cost was not taken into account in their model $[2,3]$. When scheduling is considered, time value of money has a crucial effect on locating the junction points in the access network for maximum NPV.

Optimization of NPV for underground mines was introduced by Lane [4] in 1988. He formulated the cash flows for the complete underground mining process. However, his theory can only be applied to underground mines with a given access geometry. In the past decade there has been some development towards maximizing the NPV in underground mines. Mixed integer programming is used in the optimization processes in [5-7]. In each paper, the optimal mine design has its own unique features influencing maximization of the NPV. The NPV is simply a measure of the time based value of the ore for given specific mine design parameters and underlying assumptions applied to a known or assumed ore body size and geometry [8]. However, different techniques have been applied for different underground mines and it is always assumed that the underground access is given. From the literature, it appears that no-one has tried to develop a generic theory which can be used for any underground mine. The recognition of the need for reliable 
optimization techniques and better algorithms for the mining industry is the main motivation for investigating this fundamental optimization problem.

We consider a tree where construction costs are associated with the edges and values are associated with the nodes and the edges are constructed in a given order. Such a tree is defined as a discounted Steiner tree $T$ which is the maximum NPV tree spanning a given set of nodes $N$ in space. The nodes in $T$ but not in $N$ are referred to as discounted Steiner points. The discounted Steiner points are added to the tree in order to maximize the NPV. We propose a discounted Steiner point algorithm to locate the discounted Steiner point when $T$ has three terminals. Such a network will be called a simple discounted Steiner tree. We define NPV by taking the values of the ore bodies, construction costs, development rate and discount rate into account. The location of a discounted Steiner point depends in particular on the time discount factor. It also depends on the order of reaching and extracting the ore resources and on the value of the mined material.

The proposed algorithm will find the optimal location of the discounted Steiner point. Once this point is known, the access to the mine can be determined by including the link between the discounted Steiner point and each of the given terminals. In general, these links should represent minimum length navigable paths between the end points. However, in order to make the model as simple and general as possible we relax the navigability conditions in this paper, and assume the links are straight line segments. A consequence of this, is that the theory and the algorithm outlined in this paper can be directly applied for underground mines where, the terminals points lie in a near-horizontal plane.

This paper consists of seven sections. Section 2 formulates and explains the optimization problem. Section 3 discusses degenerate cases of the location of the discounted Steiner point. Section 4 introduces the discounted Steiner point algorithm to solve the problem. The uniqueness of the discounted Steiner point and convergence of the algorithm are discussed in Section 5. Section 6 investigates the performance of the discounted Steiner point algorithm. Finally, Section 7 contains the conclusions and future research work.

\section{Problem formulation}

In this section, we model underground mine access as a discounted Steiner tree. This is a physical design problem and the parameters: locations of ore deposits and their values, development rate $D$ m p.a., cost rate $C \$ / \mathrm{m}$ and the discount rate $d \%$ p.a. are assumed to be given. In real situations, there will be additional constraints such as a gradient constraint which defines a safe climbing limit for trucks. In this paper we study the unconstrained problem without considering the more complex constrained problem.

Let $p_{0}=\left(x_{0}, y_{0}, z_{0}\right), p_{1}=\left(x_{1}, y_{1}, z_{1}\right)$ and $p_{2}=\left(x_{2}, y_{2}, z_{2}\right)$ be three given terminal points as shown in Fig. 1. The point $p_{0}$ is the surface portal or breakout point from existing infrastructure. The points $p_{1}$ and $p_{2}$ represent draw points for resources (ore deposits) with values $\$ V_{1}$ and $\$ V_{2}$, respectively. The aim is to locate the discounted Steiner point $s$ with coordinates $(x, y, z)$ to maximize the NPV.

The line segments $p_{0} s, s p_{1}$ and $s p_{2}$ are the edges in the tree. In fact the edges represent the decline links of the underground mine. The order of construction of the edges is given. First the $p_{0} s$ edge, then the $s p_{1}$ edge and finally the $s p_{2}$ 


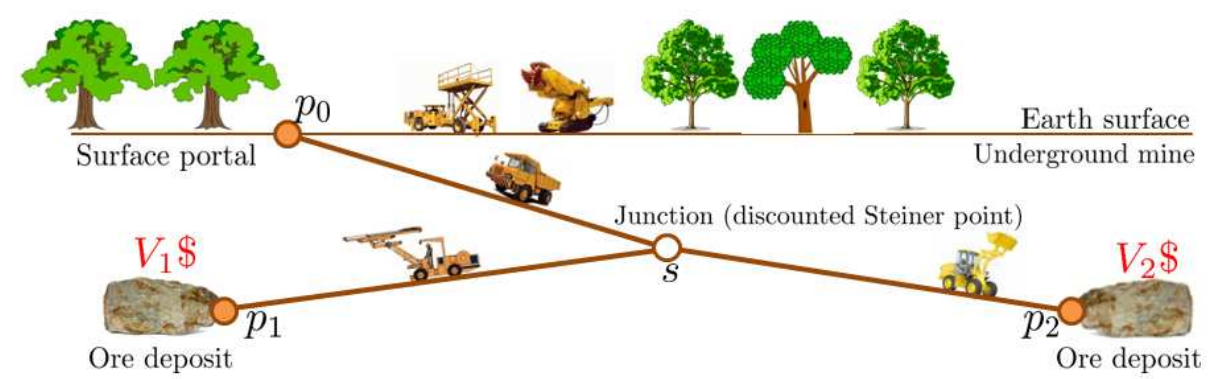

Fig. 1 A schematic representation of a simple underground mine

edge is constructed. The $s p_{1}$ edge is constructed first as it is assumed $\$ V_{1}>\$ V_{2}$. Theorem 1 provides a formula for the discounted cost for constructing an edge of length $l$ metres. We define $r=1+d, V_{c}=C D / \ln r, k=V_{1} \ln r /\left(V_{2} \ln r+C D\right)$ and use them throughout this paper.

Theorem 1 The discounted cost of constructing an edge of length $l$ is expressed as,

$$
\text { Construction cost of the edge }=V_{c}\left(1-r^{-l / D}\right)
$$

Proof The time taken to construct a portion of the edge of length $x$ is $x / D$. Therefore, the discount factor is $(1+d)^{-x / D}$. The discounted cost of developing the edge can be written,

$$
\begin{aligned}
\text { Construction cost of the edge } & =\int_{0}^{l} C(1+d)^{-x / D} d x=\frac{C D}{\ln r}\left(1-r^{-l / D}\right) \\
& =V_{c}\left(1-r^{-l / D}\right)
\end{aligned}
$$

The Net Present Value is defined by the cash flows generated from the resource extraction and construction costs of the access network. Since the construction is a cost, cash flows generated from access construction have a negative value. Finally, the NPV is the sum of all the cash flows. We do not consider other fixed costs in this model. The lengths $l_{0}, l_{1}$ and $l_{2}$ are construction lengths from $p_{0}, p_{1}$, and $p_{2}$ to $s$, respectively which are given by Euclidean distances.

Lemma 1 In a simple discounted Steiner tree, the cash flows generated from access construction are given by $N P V_{\text {con }}$ where,

$$
N P V_{\text {con }}=V_{c}\left(r^{-\left(l_{0}+l_{1}+l_{2}\right) / D}-1\right)
$$

Proof In order to construct the entire tree, the edges $p_{0} s, s p_{1}$ and $s p_{2}$ need to be constructed. Therefore, the discounted costs for constructing the entire tree are,

$$
\begin{aligned}
\text { Construction costs for the entire tree } & =\int_{0}^{l_{0}+l_{1}+l_{2}} C(1+d)^{-x / D} d x \\
& =\frac{C D}{\ln r}\left(1-r^{-\left(l_{0}+l_{1}+l_{2}\right) / D}\right)
\end{aligned}
$$


The cash flows generated from the access construction are the negative of the above and are given by $N P V_{\text {con }}$ where,

$$
N P V_{\text {con }}=V_{c}\left(r^{-\left(l_{0}+l_{1}+l_{2}\right) / D}-1\right)
$$

Lemma 2 In a simple discounted Steiner tree, the cash flows generated from resource extraction are given by $N P V_{\text {ext }}$ where,

$$
N P V_{e x t}=V_{1} r^{-\left(l_{0}+l_{1}\right) / D}+V_{2} r^{-\left(l_{0}+l_{1}+l_{2}\right) / D}
$$

Proof The resource (ore) at point $p_{1}$ is extracted before $p_{2}$ since the $s p_{1}$ edge is constructed before the $s p_{2}$ edge. Therefore, the total times taken to reach $p_{1}$ and $p_{2}$ are $t_{0}+t_{1}$ and $t_{0}+t_{1}+t_{2}$, respectively where, $t_{0}=l_{0} / D, t_{1}=l_{1} / D, t_{2}=l_{2} / D$. The NPV generated from the ore extraction is expressed as $N P V_{\text {ext }}$ where,

$$
N P V_{\text {ext }}=V_{1} r^{-\left(t_{0}+t_{1}\right)}+V_{2} r^{-\left(t_{0}+t_{1}+t_{2}\right)}
$$

Substituting the expressions for $t_{0}, t_{1}$ and $t_{2}$ into (3) gives the result.

Theorem 2 In a simple discounted Steiner tree, the NPV is given by,

$$
N P V=V_{1} r^{-\left(l_{0}+l_{1}\right) / D}+\left(V_{2}+V_{c}\right) r^{-\left(l_{0}+l_{1}+l_{2}\right) / D}-V_{c}
$$

Proof We consider only the cash flows generated from resource extraction and access construction because these directly depend on the location of the discounted Steiner point. Therefore, the total NPV is expressed as,

$$
N P V=N P V_{\text {ext }}+N P V_{\text {con }}
$$

Substituting the expressions from (1) and (2) into (5) gives the result.

The lengths $l_{0}, l_{1}$ and $l_{2}$ are functions of the discounted Steiner point coordinates $x, y$ and $z$. Equation (4) is the objective function for the problem of optimally locating the discounted Steiner point. Therefore, the problem we will analyse can be expressed as the following optimization problem;

$$
\begin{gathered}
\text { maximize } V_{1} r^{-\left(l_{0}+l_{1}\right) / D}+\left(V_{2}+V_{c}\right) r^{-\left(l_{0}+l_{1}+l_{2}\right) / D}-V_{c} \\
\text { such that } l_{0}, l_{1}, l_{2} \geq 0
\end{gathered}
$$

where $V_{1}, V_{2}, V_{c}, D$ and $r$ are constants. The decision variables are $l_{0}, l_{1}$ and $l_{2}$ and they are functions of the coordinates of the discounted Steiner point, $x, y$ and $z$. There will be additional constraints on $l_{0}, l_{1}$ and $l_{2}$ that are imposed by the geometry of the network. Later, we will obtain such a constraint which is given by (13).

A maximum always exists since the NPV is bounded above by $V_{1}+V_{2}$. It is obvious that, if the discounted Steiner point is not on the plane defined by the three vertices, then the NPV can be increased by moving the discounted Steiner point towards and perpendicular to the plane, since this decreases $l_{0}, l_{1}$ and $l_{2}$. Therefore, the discounted Steiner point always lies in the interior or on the boundary of the triangle given by the three vertices $p_{0}, p_{1}$ and $p_{2}$. 
The objective function is differentiable on the problem domain with respect to $x, y$ and $z$ except at $p_{0}, p_{1}$ and $p_{2}$ since these points are degenerate Steiner points. We will discuss these cases further in Section 3.

The problem is a non-convex problem, therefore methods that assume convexity cannot be used. The optimal point which gives the maximum NPV is found by differentiating the objective function and setting this equal to zero.

$$
\begin{aligned}
\frac{\partial N P V}{\partial x}= & \frac{-V_{1} \ln r}{D} r^{-\left(l_{0}+l_{1}\right) / D}\left(\frac{\partial l_{0}}{\partial x}+\frac{\partial l_{1}}{\partial x}\right) \\
& -\left(\frac{V_{2} \ln r}{D}+C\right) r^{-\left(l_{0}+l_{1}+l_{2}\right) / D}\left(\frac{\partial l_{0}}{\partial x}+\frac{\partial l_{1}}{\partial x}+\frac{\partial l_{2}}{\partial x}\right) \\
= & 0
\end{aligned}
$$

Therefore,

$$
\frac{V_{1} \ln r}{D}\left(\frac{\partial l_{0}}{\partial x}+\frac{\partial l_{1}}{\partial x}\right)+\left(\frac{V_{2} \ln r}{D}+C\right) r^{-l_{2} / D}\left(\frac{\partial l_{0}}{\partial x}+\frac{\partial l_{1}}{\partial x}+\frac{\partial l_{2}}{\partial x}\right)=0
$$

Similarly, differentiating the objective function with respect to $y$ and $z$ and setting these equal to 0 , we obtain,

$$
(A+B) \nabla\left(l_{0}+l_{1}\right)+B \nabla l_{2}=0
$$

where $A=V_{1} \ln r / D$ and $B=\left(V_{2} \ln r / D+C\right) r^{-l_{2} / D}$.

Next, we develop an important property of the angles at the discounted Steiner point in a simple discounted Steiner tree.

Theorem 3 If the discounted Steiner point does not coincide with $p_{0}, p_{1}$ or $p_{2}$, then the paths from the discounted Steiner point to $p_{0}$ and $p_{1}$ make equal angles with the path from the discounted Steiner point to $p_{2}$ and that angle $\theta$ is expressed as,

$$
\cos \theta=-\frac{1}{2\left(k r^{l_{2} / D}+1\right)}
$$

Proof Let $\mathbf{u}_{0}, \mathbf{u}_{1}$ and $\mathbf{u}_{2}$ be the unit vectors which are directed from the fixed points $p_{0}, p_{1}$, and $p_{2}$, respectively towards the discounted Steiner point. Let $\theta$ be the angle between $\mathbf{u}_{1}$ and $\mathbf{u}_{2}$ and $\theta^{\prime}$ be the angle between $\mathbf{u}_{0}$ and $\mathbf{u}_{2}$ as shown in Fig. 2. The unit vectors are expressed as the gradients $\mathbf{u}_{0}=\nabla l_{0}, \mathbf{u}_{1}=\nabla l_{1}$ and $\mathbf{u}_{2}=\nabla l_{2}$.

Substituting the values of $\nabla l_{0}, \nabla l_{1}$ and $\nabla l_{2}$ into (6),

$$
(A+B)\left(\mathbf{u}_{0}+\mathbf{u}_{1}\right)+B \mathbf{u}_{2}=0
$$

By taking the dot product of (8) with $\left(\mathbf{u}_{0}-\mathbf{u}_{1}\right)$,

$$
(A+B)\left(\mathbf{u}_{0}+\mathbf{u}_{1}\right) \cdot\left(\mathbf{u}_{0}-\mathbf{u}_{1}\right)+B \mathbf{u}_{2} \cdot\left(\mathbf{u}_{0}-\mathbf{u}_{1}\right)=0
$$

Since $\left(\mathbf{u}_{0}+\mathbf{u}_{1}\right) \cdot\left(\mathbf{u}_{0}-\mathbf{u}_{1}\right)=\left|\mathbf{u}_{0}\right|^{2}-\left|\mathbf{u}_{1}\right|^{2}=1-1=0$, the expression above reduces to,

$$
\begin{aligned}
\mathbf{u}_{2} \cdot \mathbf{u}_{0} & =\mathbf{u}_{2} \cdot \mathbf{u}_{1} \\
\left|\mathbf{u}_{2}\right|\left|\mathbf{u}_{0}\right| \cos \theta & =\left|\mathbf{u}_{2}\right|\left|\mathbf{u}_{1}\right| \cos \theta^{\prime} \\
\cos \theta & =\cos \theta^{\prime} \\
\theta & =\theta^{\prime}
\end{aligned}
$$




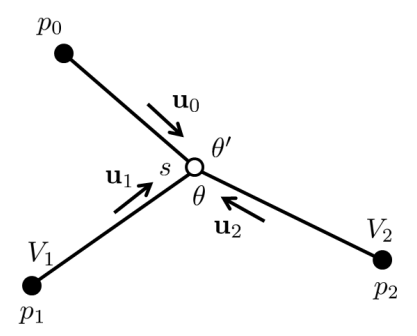

Fig. 2 The vector representation of the problem

By taking the dot product of (8) with $\mathbf{u}_{2}$,

$$
(A+B)\left(\mathbf{u}_{0}+\mathbf{u}_{1}\right) \cdot \mathbf{u}_{2}+B \mathbf{u}_{2} \cdot \mathbf{u}_{2}=0
$$

Since $\mathbf{u}_{0} \cdot \mathbf{u}_{2}=\mathbf{u}_{1} \cdot \mathbf{u}_{2}=\cos \theta$ and $\mathbf{u}_{2} \cdot \mathbf{u}_{2}=1$, by substituting these values into (9),

$$
\begin{array}{r}
2(A+B) \cos \theta+B=0 \\
\cos \theta=-\frac{1}{2(A / B+1)}=-\frac{1}{2\left(k r^{l_{2} / D}+1\right)}
\end{array}
$$

Lemmas 3 and 4 provide the upper and lower bounds of the angle $\theta$ which are defined for zero and infinite discount rates, respectively.

Lemma 3 If the operating discount rate is zero, then the discounted Steiner point coincides with the classical Steiner point.

Proof If $d=0$, then $r=1$ and $k=0$. Substituting the values of $r$ and $k$ into (7),

$$
\begin{array}{r}
\cos \theta=-\frac{1}{2\left(k r^{l_{2} / D}+1\right)}=-1 / 2 \\
\theta=2 \pi / 3
\end{array}
$$

which corresponds to the classical Steiner position and this angle is the upper bound for the angle $\theta$.

Lemma 4 For the limiting case of an infinite discount rate the discounted Steiner point is at the intersection of the line connecting $p_{0}$ and $p_{1}$ and the line perpendicular to it and passing through $p_{2}$.

Proof If $d \rightarrow \infty$, then $r \rightarrow \infty$. By substituting the limiting value of $r$ into (7),

$$
\begin{array}{r}
\lim _{r \rightarrow \infty} \cos \theta=\lim _{r \rightarrow \infty}-\frac{1}{2\left(k r^{l_{2} / D}+1\right)}=0 \\
\lim _{r \rightarrow \infty} \theta=\pi / 2
\end{array}
$$

Hence, $\pi / 2$ is the lower bound of angle $\theta$.

Therefore, from Lemmas 3 and 4 , for any discount rate between 0 and $\infty$, the range of an optimal $\theta$ is $\pi / 2<\theta<2 \pi / 3$. 
Corollary 1 If the operating discount rate is zero, then the problem of maximizing the NPV is reduced to minimizing the total length $l_{0}+l_{1}+l_{2}$.

Proof By expanding the objective function in (4),

$$
N P V=V_{1} r^{-\left(l_{0}+l_{1}\right) / D}+V_{2} r^{-\left(l_{0}+l_{1}+l_{2}\right) / D}+\frac{C D}{\ln r}\left(r^{-\left(l_{0}+l_{1}+l_{2}\right) / D}-1\right)
$$

If $d=0$, then $r=1$ so the first two terms reduce to $V_{1}+V_{2}$ and the third term is an indeterminate form as $r$ approaches 1. Therefore, by using l'Hôpital's rule [9] for the third term,

$$
\lim _{r \rightarrow 1} \frac{C D}{\ln r}\left(r^{-\left(l_{0}+l_{1}+l_{2}\right) / D}-1\right)=-C\left(l_{0}+l_{1}+l_{2}\right)
$$

By substituting the values above into (10), the new NPV function becomes $N P V_{(d=0)}$, where

$$
N P V_{(d=0)}=V_{1}+V_{2}-C\left(l_{0}+l_{1}+l_{2}\right)
$$

which implies,

$$
\max \left[V_{1}+V_{2}-C\left(l_{0}+l_{1}+l_{2}\right)\right] \equiv \min \left[C\left(l_{0}+l_{1}+l_{2}\right)-V_{1}-V_{2}\right]
$$

Therefore, the problem is reduced to a length minimization problem when the operating discount rate is zero and the solution is indeed the classical Steiner point.

So far, we have developed one equation in terms of $\theta$ and $l_{2}$, Equation (7), using the objective function. Now, we relate $\theta$ and $l_{2}$ geometrically and generate a second equation, Equation (13), in terms of $\theta$ and $l_{2}$.

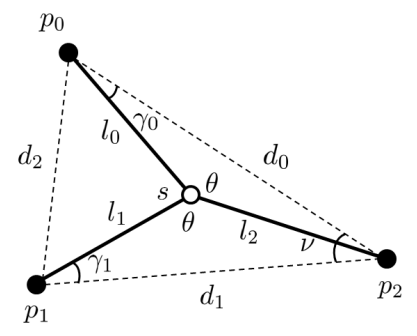

Fig. 3 The geometric representation of the problem

Let $\gamma_{0}=\angle s p_{0} p_{2}, \gamma_{1}=\angle s p_{1} p_{2}, \nu=\angle p_{0} p_{2} p_{1}$ and $d_{0}, d_{1}, d_{2}$ be the given Euclidean distances as shown in Fig. 3. By applying the cosine rule to triangle $p_{0} p_{1} p_{2}$,

$$
\nu=\arccos \left(\frac{d_{0}^{2}+d_{1}^{2}-d_{2}^{2}}{2 d_{0} d_{1}}\right)
$$


By applying the sine rule to triangles $p_{0} p_{2} s$ and $p_{1} p_{2} s$,

$$
\sin \gamma_{0}=\frac{l_{2} \sin \theta}{d_{0}} \quad \sin \gamma_{1}=\frac{l_{2} \sin \theta}{d_{1}}
$$

Summing the angles in the quadrilateral $p_{0} p_{2} p_{1} s$ yields:

$$
\gamma_{0}+\nu+\gamma_{1}+2 \theta=2 \pi
$$

By substituting the values for $\gamma_{1}$ and $\gamma_{0}$ into (12), $l_{2}$ can be obtained, where

$$
l_{2}=\frac{d_{0} d_{1}|\sin (2 \theta+\nu)|}{\sin \theta \sqrt{d_{0}^{2}+d_{1}^{2}+2 \cos (2 \theta+\nu) d_{0} d_{1}}}
$$

since $\sin (2 \theta+\nu)<0$.

\section{Degenerate cases of the discounted Steiner point}

Next, we consider the degenerate cases where the discounted Steiner point coincides with one of the vertices $p_{0}, p_{1}$ or $p_{2}$. Equation $(7)$ is used to calculate some special angles for which the discounted Steiner point is located at $p_{0}, p_{1}$ and $p_{2}$. These angles are called critical angles. For each vertex the critical angle is the minimum angle for which the discounted Steiner point coincides with the vertex. The critical angle determines whether the discounted Steiner point lies at $p_{0}, p_{1}$ or $p_{2}$.

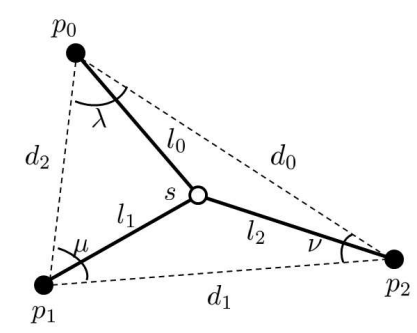

Fig. 4 The discounted Steiner point degenerate cases

Let $\lambda, \mu$ and $\nu$ be the angles $\angle p_{1} p_{0} p_{2}, \angle p_{0} p_{1} p_{2}$ and $\angle p_{1} p_{2} p_{0}$, respectively. By applying the cosine rule for triangle $p_{0} p_{1} p_{2}$ in Fig. 4 ,

$$
\begin{aligned}
& \lambda=\arccos \left(\frac{d_{0}^{2}+d_{2}^{2}-d_{1}^{2}}{2 d_{0} d_{2}}\right) \\
& \mu=\arccos \left(\frac{d_{1}^{2}+d_{2}^{2}-d_{0}^{2}}{2 d_{1} d_{2}}\right)
\end{aligned}
$$

By substituting the values of $d_{0}, d_{1}$ and $d_{2}$ into (11), (14) and (15) the angles $\nu$, $\lambda$, and $\mu$ can be found respectively.

Let $\varphi, \omega$ and $\psi$ be the critical angles for which the discounted Steiner point lies at $p_{0}, p_{1}$ and $p_{2}$, respectively. The discounted Steiner point coincides with a vertex if the angle at the vertex is greater than or equal to the critical angle. 
Lemma 5 If $\nu \geq \psi$, then the discounted Steiner point coincides with $p_{2}$ and

(I) the critical angle $\psi$ is given by,

$$
\psi=2 \arccos \left(\frac{1}{2(k+1)}\right)
$$

(II) the maximum $N P V$ is given by $N P V_{\left(s=p_{2}\right)}$, where

$$
N P V_{\left(s=p_{2}\right)}=\left(V_{1}+V_{2}+V_{c}\right) r^{-\left(d_{0}+d_{1}\right) / D}-V_{c}
$$

Proof The conditions that need to be satisfied so that the discounted Steiner point is located at $p_{2}$ are $l_{2}=0$ and $2 \pi-2 \theta=\psi$. By substituting these values into (7),

$$
\begin{gathered}
\cos \left(\pi-\frac{\psi}{2}\right)=-\frac{1}{2(k+1)} \\
\psi=2 \arccos \left(\frac{1}{2(k+1)}\right)
\end{gathered}
$$

Therefore, if $\nu \geq \psi$ the discounted Steiner point coincides with $p_{2}$. When the discounted Steiner point is at $p_{2}, l_{0}=d_{0}, l_{1}=d_{1}$ and $l_{2}=0$. Substituting these values into (4) gives the resultant NPV.

Equation (16) can be verified for a special case, where if $d=0$, then $r=1$ and $k=0$ so $\psi$ should be $2 \pi / 3$ as for the classical Steiner tree. By substituting the value of $k$ into (16),

$$
\psi=2 \arccos \frac{1}{2}=\frac{2 \pi}{3}
$$

By substituting $k=V_{1} \ln r /\left(V_{2} \ln r+C D\right)$ into (16),

$$
\psi=2 \arccos \left(\frac{V_{2} \ln r+C D}{2\left(V_{1} \ln r+V_{2} \ln r+C D\right)}\right)
$$

Note that $V_{1} \ln r>0$. Therefore, $\psi \geq 2 \pi / 3$ in general.

Lemma 6 If $\mu \geq \omega$, then the discounted Steiner point coincides with $p_{1}$ and

(I) the critical angle $\omega$ is given by,

$$
\omega=\arccos \left(\frac{-1}{2\left(k r^{d_{1} / D}+1\right)}\right)
$$

(II) the maximum $N P V$ is given by $N P V_{\left(s=p_{1}\right)}$, where

$$
N P V_{\left(s=p_{1}\right)}=V_{1} r^{-d_{2} / D}+\left(V_{2}+V_{c}\right) r^{-\left(d_{1}+d_{2}\right) / D}-V_{c}
$$

Proof The conditions that need to be satisfied so that the discounted Steiner point is located at $p_{1}$ are $l_{2}=d_{1}$ and $\theta=\omega$. By substituting these values into (7),

$$
\begin{array}{r}
\cos \omega=\frac{-1}{2\left(k r^{d_{1} / D}+1\right)} \\
\omega=\arccos \left(\frac{-1}{2\left(k r^{d_{1} / D}+1\right)}\right)
\end{array}
$$

Hence, if $\mu \geq \omega$ the discounted Steiner point lies at point $p_{1}$. When the discounted Steiner point is at $p_{1}, l_{0}=d_{2}, l_{1}=0$ and $l_{2}=d_{1}$. Substituting these values into (4) gives the resultant NPV. 
Equation (18) can be verified as before for the classical Steiner tree case. If $d=0$, then $r=1$ and $k=0$. By substituting the value of $k$ into (18),

$$
\omega=\arccos \frac{-1}{2}=\frac{2 \pi}{3}
$$

Similar to the result in Lemma $5, \omega \leq 2 \pi / 3$ in general.

Lemma 7 If $\lambda \geq \varphi$, then the discounted Steiner point coincides with $p_{0}$ and

(I) the critical angle $\varphi$ is given by,

$$
\varphi=\arccos \left(\frac{-1}{2\left(k r^{d_{0} / D}+1\right)}\right)
$$

(II) the maximum $N P V$ is given by $N P V_{\left(s=p_{0}\right)}$, where

$$
N P V_{\left(s=p_{0}\right)}=V_{1} r^{-d_{2} / D}+\left(V_{2}+V_{c}\right) r^{-\left(d_{0}+d_{2}\right) / D}-V_{c}
$$

Proof The conditions that need to be satisfied so that the discounted Steiner point is located at $p_{0}$ are $l_{2}=d_{0}$ and $\theta=\varphi$. By substituting these values into (7),

$$
\begin{array}{r}
\cos \varphi=\frac{-1}{2\left(k r^{d_{0} / D}+1\right)} \\
\varphi=\arccos \left(\frac{-1}{2\left(k r^{d_{0} / D}+1\right)}\right)
\end{array}
$$

Therefore, if $\lambda \geq \varphi$ the discounted Steiner point lies at point $p_{0}$. Similarly, when the discounted Steiner point is at $p_{0}, l_{0}=0, l_{1}=d_{2}$ and $l_{2}=d_{0}$. Substituting these values into (4) the resultant NPV can be obtained.

Equation (20) can be verified for a special case as before, where if $d=0$, then $r=1$ and $k=0$. By substituting the value of $k$ into (20),

$$
\varphi=\arccos \frac{-1}{2}=\frac{2 \pi}{3}
$$

Similar to Lemma $5, \varphi \leq 2 \pi / 3$ in general.

For a particular given example, first $\psi, \omega$ and $\varphi$ should be calculated and compared with $\lambda, \mu$ and $\nu$. If one of the inequalities $\nu \geq \psi, \mu \geq \omega$ or $\lambda \geq \varphi$ is satisfied, then the optimal location of the discounted Steiner point will be at one of the vertices.

\section{Discounted Steiner Point Algorithm (DSPA)}

In Section 2, (7) and (13) were developed in terms of $\theta$ and $l_{2}$. In this section, an iterative process is presented to find the optimal values for $\theta$ and $l_{2}$. If these two parameters are known, the discounted Steiner point coordinates can be calculated by solving three quadratic simultaneous equations.

The discounted Steiner point algorithm is a fixed point iteration algorithm where we want to solve for $\theta$. It seeks the solution by an iterative process such that $\theta_{n+1}=f\left(\theta_{n}\right)$ starting from some initial value $\theta_{0}$. 


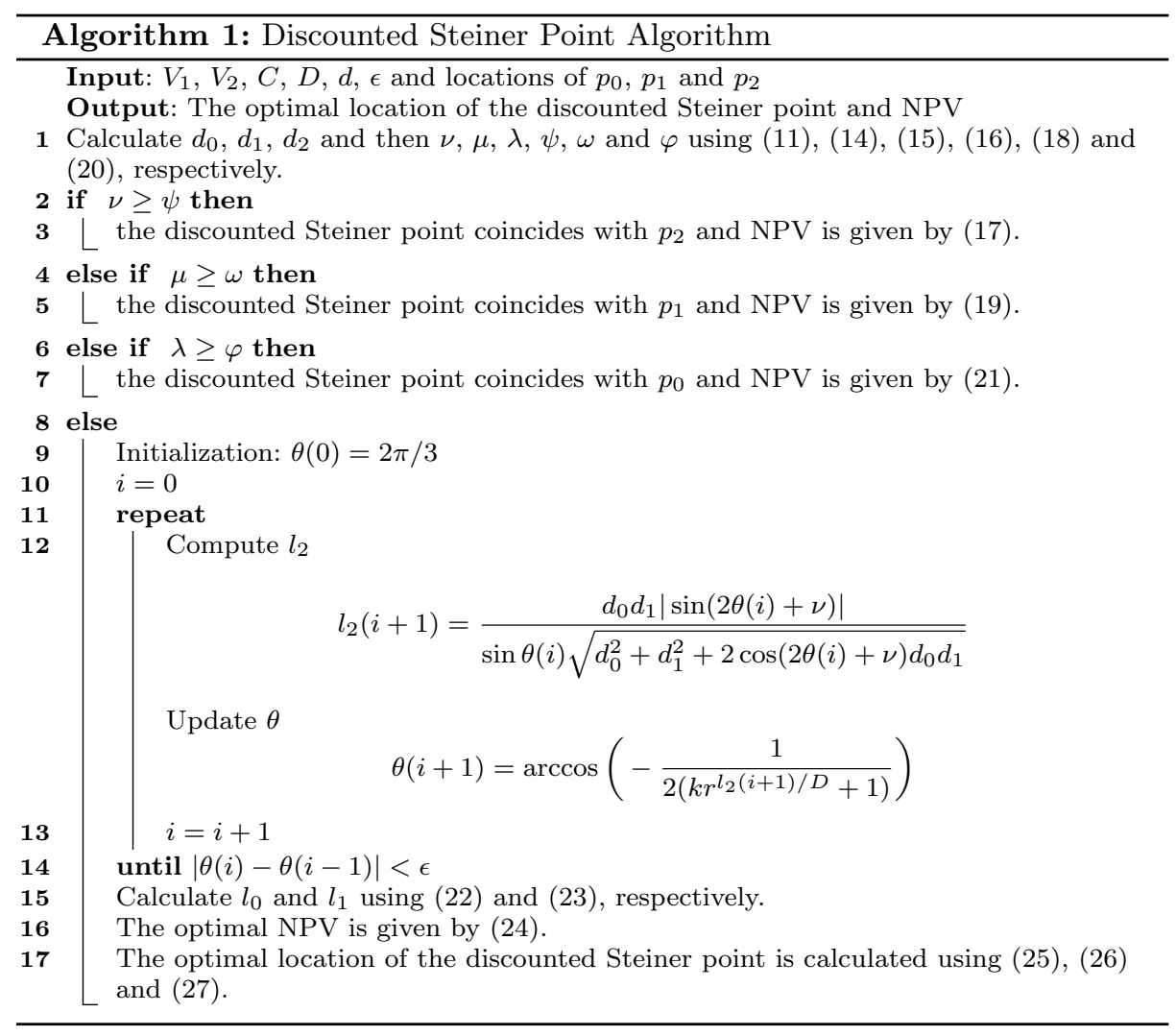

Let $\hat{\theta}$ and $\hat{l}_{2}$ be the optimal values obtained from the iterative process. Then $\hat{l}_{0}$ and $\hat{l}_{1}$ are calculated by applying the sine rule for triangles $p_{0} s p_{2}$ and $p_{1} s p_{2}$ in Fig. 3.

$$
\begin{aligned}
& \hat{l}_{0}=\frac{d_{0} \sin \left(\hat{\theta}+\gamma_{0}\right)}{\sin \hat{\theta}} \\
& \hat{l}_{1}=\frac{d_{1} \sin \left(\hat{\theta}+\gamma_{1}\right)}{\sin \hat{\theta}}
\end{aligned}
$$

The maximum NPV is expressed as $N P V^{*}$, where

$$
N P V^{*}=V_{1} r^{-\left(\hat{l}_{0}+\hat{l}_{1}\right) / D}+\left(V_{2}+V_{c}\right) r^{-\left(\hat{l}_{0}+\hat{l}_{1}+\hat{l}_{2}\right) / D}-V_{c}
$$

Since $\hat{l}_{0}, \hat{l}_{1}$ and $\hat{l}_{2}$ are known, $x, y, z$ can be calculated by solving three quadratic simultaneous equations (25), (26) and (27).

$$
\begin{aligned}
& \hat{l}_{0}^{2}=\left(x_{0}-x\right)^{2}+\left(y_{0}-y\right)^{2}+\left(z_{0}-z\right)^{2} \\
& \hat{l}_{1}^{2}=\left(x-x_{1}\right)^{2}+\left(y-y_{1}\right)^{2}+\left(z-z_{1}\right)^{2} \\
& \hat{l}_{2}^{2}=\left(x-x_{2}\right)^{2}+\left(y-y_{2}\right)^{2}+\left(z-z_{2}\right)^{2}
\end{aligned}
$$


The iterative process converges to the optimal solution very quickly and convergence is independent of the initial value of $\theta$. The uniqueness and convergence will be established in the next section.

\section{Uniqueness of the discounted Steiner point and the convergence of the algorithm}

We investigate the problem of determining conditions for the discounted Steiner point to be unique and for the algorithm to converge. The DSPA is a fixed point iteration of the function,

$$
f(\theta)=\arccos \left(-\frac{1}{2\left(k r^{l_{2} / D}+1\right)}\right)
$$

where $l_{2}$ is a function of $\theta$ and is given by (13).

Previously we have shown that $\pi / 2<\theta<2 \pi / 3$ but it also needs to be established for the $\theta$ values at each iteration. Let $f(\theta)=\arccos (-1 / x)$ where $x$ is a function of $\theta$ and is given by $x=2\left(k r^{l_{2} / D}+1\right)$. Since $x>2$, it follows that $-1 / 2<-1 / x<0$ and so $\pi / 2<f(\theta)<2 \pi / 3$. Therefore, the $n^{\text {th }}$ iterate, $\theta_{n}$, satisfies $\pi / 2<\theta_{n}<2 \pi / 3$ because $\theta_{n}=f\left(\theta_{n-1}\right)$.

It is sufficient to find a condition ensuring that $\left|f^{\prime}(\theta)\right|<1$ which establishes the uniqueness of the discounted Steiner point and the convergence of the algorithm. Since $d x / d l_{2}=2 k r^{l_{2} / D} \ln r / D=(x-2) \ln r / D$,

$$
f^{\prime}(\theta)=-\frac{1 / x^{2}}{\sqrt{1-1 / x^{2}}} \frac{d x}{d l_{2}} \frac{d l_{2}}{d \theta}=-\frac{1}{x \sqrt{x^{2}-1}} \frac{d x}{d l_{2}} \frac{d l_{2}}{d \theta}=-\frac{\ln r}{D} \frac{x-2}{x \sqrt{x^{2}-1}} \frac{d l_{2}}{d \theta}
$$

Next, we will find a bound for $d l_{2} / d \theta$. For convenience, let $v=\left(d_{0}^{2}+d_{1}^{2}\right) / d_{0} d_{1}+$ $2 \cos (2 \theta+\nu)$. Then,

$$
l_{2}=-\frac{\sqrt{d_{0} d_{1}} \sin (2 \theta+\nu)}{v^{1 / 2} \sin \theta}
$$

and,

$$
\frac{d v}{d \theta}=-4 \sin (2 \theta+\nu)
$$

Differentiating (29) with respect to $\theta$ yields,

$$
\frac{d l_{2}}{d \theta}=-\sqrt{d_{0} d_{1}} w
$$

where

$$
w=\frac{2 v^{1 / 2} \sin \theta \cos (2 \theta+\nu)-\sin (2 \theta+\nu)\left[v^{1 / 2} \cos \theta+\left(v^{-1 / 2} / 2\right)(d v / d \theta) \sin \theta\right]}{v \sin ^{2} \theta}
$$


If we can find an upper bound, $M$, for $w$, then $w \leq M$ and so $d l_{2} / d \theta \geq$ $-\sqrt{d_{0} d_{1}} M$.

$$
\begin{aligned}
w= & \frac{2 v \sin \theta \cos (2 \theta+\nu)-\sin (2 \theta+\nu)[v \cos \theta-2 \sin (2 \theta+\nu) \sin \theta]}{v^{3 / 2} \sin ^{2} \theta} \\
= & \frac{2 \sin \theta \cos (2 \theta+\nu)-\sin (2 \theta+\nu) \cos \theta}{v^{1 / 2} \sin ^{2} \theta}+\frac{2 \sin ^{2}(2 \theta+\nu) \sin \theta}{v^{3 / 2} \sin ^{2} \theta} \\
= & \frac{2 \sin \theta\left[\cos ^{2}(\theta+\nu / 2)-\sin ^{2}(\theta+\nu / 2)\right]-2 \sin (\theta+\nu / 2) \cos (\theta+\nu / 2) \cos \theta}{v^{1 / 2} \sin ^{2} \theta} \\
& +\frac{8 \sin ^{2}(\theta+\nu / 2) \cos ^{2}(\theta+\nu / 2) \sin \theta}{v^{3 / 2} \sin ^{2} \theta}
\end{aligned}
$$

Now,

$$
v=\left(d_{0}-d_{1}\right)^{2} / d_{0} d_{1}+2+2 \cos (2 \theta+\nu) \geq 2[1+\cos (2 \theta+\nu)]=4 \cos ^{2}(\theta+\nu / 2)
$$

Therefore, using the fact that $\sin \theta>0$ because $\pi / 2<\theta<2 \pi / 3$,

$$
\begin{aligned}
w \leq & \frac{2 \sin \theta\left[\cos ^{2}(\theta+\nu / 2)-\sin ^{2}(\theta+\nu / 2)\right]-2 \sin (\theta+\nu / 2) \cos (\theta+\nu / 2) \cos \theta}{v^{1 / 2} \sin ^{2} \theta} \\
& +\frac{8 \sin ^{2}(\theta+\nu / 2) \cos ^{2}(\theta+\nu / 2) \sin \theta}{4 v^{1 / 2} \cos ^{2}(\theta+\nu / 2) \sin ^{2} \theta} \\
= & \frac{2 \sin \theta \cos ^{2}(\theta+\nu / 2)-2 \sin \theta \sin ^{2}(\theta+\nu / 2)-2 \sin (\theta+\nu / 2) \cos (\theta+\nu / 2) \cos \theta}{v^{1 / 2} \sin ^{2} \theta} \\
& +\frac{2 \sin ^{2}(\theta+\nu / 2) \sin \theta}{v^{1 / 2} \sin ^{2} \theta} \\
= & \frac{2 \sin \theta \cos ^{2}(\theta+\nu / 2)-2 \sin (\theta+\nu / 2) \cos (\theta+\nu / 2) \cos \theta}{v^{1 / 2} \sin ^{2} \theta} \\
= & \frac{2 \cos (\theta+\nu / 2)[\sin \theta \cos (\theta+\nu / 2)-\cos \theta \sin (\theta+\nu / 2)]}{v^{1 / 2} \sin ^{2} \theta} \\
= & \frac{-2 \cos (\theta+\nu / 2) \sin (\nu / 2)}{v^{1 / 2} \sin ^{2} \theta}
\end{aligned}
$$

Since $\theta>\pi / 2, \nu>0$, and $2 \theta$ and $\nu$ are two of the angles in the quadrilateral $p_{0} s p_{1} p_{2}$, it follows that $\pi<2 \theta+\nu<2 \pi$. Thus, $\pi / 2<\theta+\nu / 2<\pi$ and so $\cos (\theta+\nu / 2)<0$. Since $v \geq 4 \cos ^{2}(\theta+\nu / 2)$, it follows that $v^{1 / 2} \geq-2 \cos (\theta+\nu / 2)$. Hence,

$$
w \leq \frac{-2 \cos (\theta+\nu / 2) \sin (\nu / 2)}{-2 \cos (\theta+\nu / 2) \sin ^{2} \theta}=\frac{\sin (\nu / 2)}{\sin ^{2} \theta} \leq \frac{4}{3}
$$

where the last inequality follows from the fact that $\pi / 2<\theta<2 \pi / 3$ and so $\sin \theta>\sqrt{3} / 2$. Therefore from (31),

$$
\frac{d l_{2}}{d \theta} \geq-\sqrt{d_{0} d_{1}} M
$$

where $M=4 / 3$ is an upper bound for $w$. Provided that $d l_{2} / d \theta<0$ (which is proved below) it follows from (28) that,

$$
\left|f^{\prime}(\theta)\right| \leq \frac{x-2}{x \sqrt{x^{2}-1}} \times \frac{4 \sqrt{d_{0} d_{1}} \ln r}{3 D}
$$


The maximum value of $(x-2) /\left(x \sqrt{x^{2}-1}\right)$ with $x>2$ occurs at the largest solution to the equation $x^{3}-4 x^{2}+2=0$. Denoting this maximum value by $\beta$, we have $\beta \approx 0.129$. Therefore,

$$
\left|f^{\prime}(\theta)\right| \leq \frac{4 \beta \sqrt{d_{0} d_{1}} \ln r}{3 D}
$$

In order to ensure that $\left|f^{\prime}(\theta)\right|<1$, we must have,

$$
\sqrt{d_{0} d_{1}}<\left(\frac{3}{4 \beta}\right) D / \ln r
$$

The numeric factor $3 / 4 \beta$ is approximately equal to 5.80 . The left hand side of (33) is the geometric mean of the distances from the surface portal to the second resource point and the first resource point to the second resource point; it can be interpreted as a length scale for the geometry of the problem. The expression $D / \ln r$ is the length of decline that would be constructed during the characteristic time for the discounting. At a typical construction rate of $2000 \mathrm{~m}$ p.a. and with a discount rate of $10 \%$ p.a., the right hand side of (33) is more than $120 \mathrm{~km}$. For non-uniqueness, inequality (33) would have to be violated meaning either $d_{0}$ or $d_{1}$ would have to be greater than $120 \mathrm{~km}$, which is unrealistic for a real underground mine. Therefore the condition (33) is easily satisfied in practice.

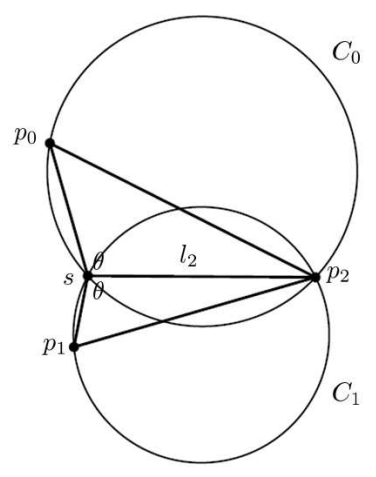

Fig. 5 Proof that $d l_{2} / d \theta<0$.

In order to complete the proof, we need to show that $d l_{2} / d \theta<0$. Let $C_{0}$ be the circle through the given points $p_{0}$ and $p_{2}$ and the Steiner point $s$, and let $C_{1}$ be the circle through $p_{1}, p_{2}$ and $s$, as shown in Fig. 5 . Since $\theta>\pi / 2$, the angles of the $\operatorname{arcs} p_{0} s p_{2}$ and $p_{1} s p_{2}$ are both less than $\pi$. Hence, the arc of $C_{0}$ from $s$ to $p_{2}$ and not passing through $p_{0}$ and the arc of $C_{1}$ from $s$ to $p_{2}$ and not passing through $p_{1}$ are minor arcs of their respective circles.

If $s$ is now moved while the circles remain fixed, then the angle $p_{0} s p_{2}$ is greater than, equal to, or less than $\theta$ according to whether $s$ is inside, on or outside $C_{0}$, and similarly for the angle $p_{1} s p_{2}$ and the circle $C_{1}$. Hence, if $s$ is moved to a location where the two angles remain equal to each other and are greater than $\theta$, then $s$ must be in the lens-shaped region where the interiors of $C_{0}$ and $C_{1}$ intersect. For $s$ within this region, the length of the line segment $s p_{2}$ is less than $l_{2}$. Therefore, $d l_{2} / d \theta<0$. 


\section{Numerical trials}

Numerical trials have been carried out to analyse the performance of the discounted Steiner point algorithm. The algorithm is applied to data values that are typical for an underground mine and then the improvement of the NPV is discussed.

The developed algorithm was applied using the two data sets below for a range of discount rates and then the optimal locations of the discounted Steiner point were obtained. The discount rates 5, 10 and $20 \%$ p.a. are the most realistic in underground mine operations.

Case 1:

$V_{1}=\$ 60 \mathrm{M}, V_{2}=\$ 40 \mathrm{M}$

$C=\$ 6000 / \mathrm{m}, D=1560 \mathrm{~m}$ p.a.

$d=0,5,10,20,50,100, \infty \%$ p.a.

$p_{0}=(200,0,1000), p_{1}=(0,0,0)$ and $p_{2}=(1000,0,0)$

Case 2:

$V_{1}=\$ 100 \mathrm{M}, V_{2}=\$ 1 \mathrm{M}$

$C=\$ 4000 / \mathrm{m}, D=2000 \mathrm{~m}$ p.a.

$d=0,5,10,20,50,100, \infty \%$ p.a.

$p_{0}=(0,0,1000), p_{1}=(600,0,0)$ and $p_{2}=(1250,0,750)$

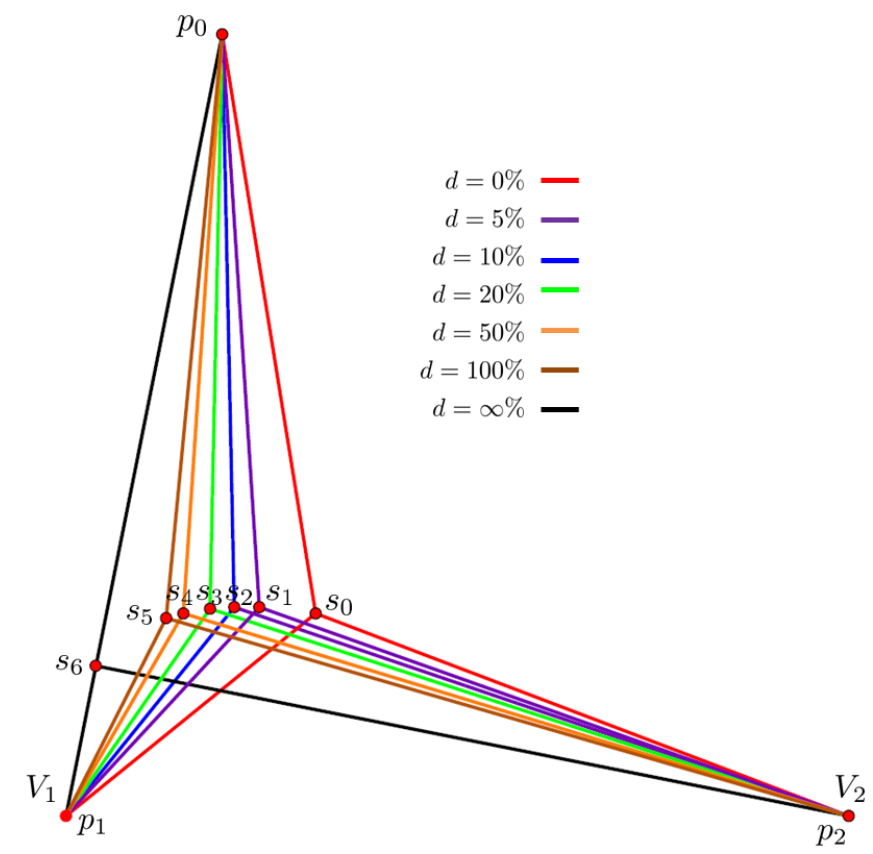

Fig. 6 The optimal locations of the discounted Steiner point for Case 1 
Table 1 Variation of the NPV for a range of discount rates for Case 1

\begin{tabular}{cccccc}
\hline $\begin{array}{c}\text { Discount } \\
\text { rate } / \% \text { p.a. }\end{array}$ & $\begin{array}{c}\text { Location } \\
\text { of the DSP }\end{array}$ & $\begin{array}{c}\theta \\
/ \mathrm{deg}\end{array}$ & $\begin{array}{c}l_{2} \\
/ \mathrm{m}\end{array}$ & $\begin{array}{c}\text { NPV } \\
/ \$ \mathrm{M}\end{array}$ & $\begin{array}{c}\text { Improvement } \\
/ \$\end{array}$ \\
\hline 0 & $s_{0}=(319,0,259)$ & 120 & 728 & 88.660 & 0 \\
5 & $s_{1}=(247,0,267)$ & 113 & 798 & 84.618 & 49000 \\
10 & $s_{2}=(215,0,267)$ & 110 & 828 & 80.997 & 159000 \\
20 & $s_{3}=(184,0,265)$ & 106 & 857 & 74.700 & 393000 \\
50 & $s_{4}=(150,0,259)$ & 103 & 888 & 60.875 & 958000 \\
100 & $s_{5}=(128,0,253)$ & 100 & 907 & 46.971 & 1516000 \\
$\infty$ & $s_{6}=(38,0,192)$ & 90 & 980 & 0 & 0 \\
\hline
\end{tabular}

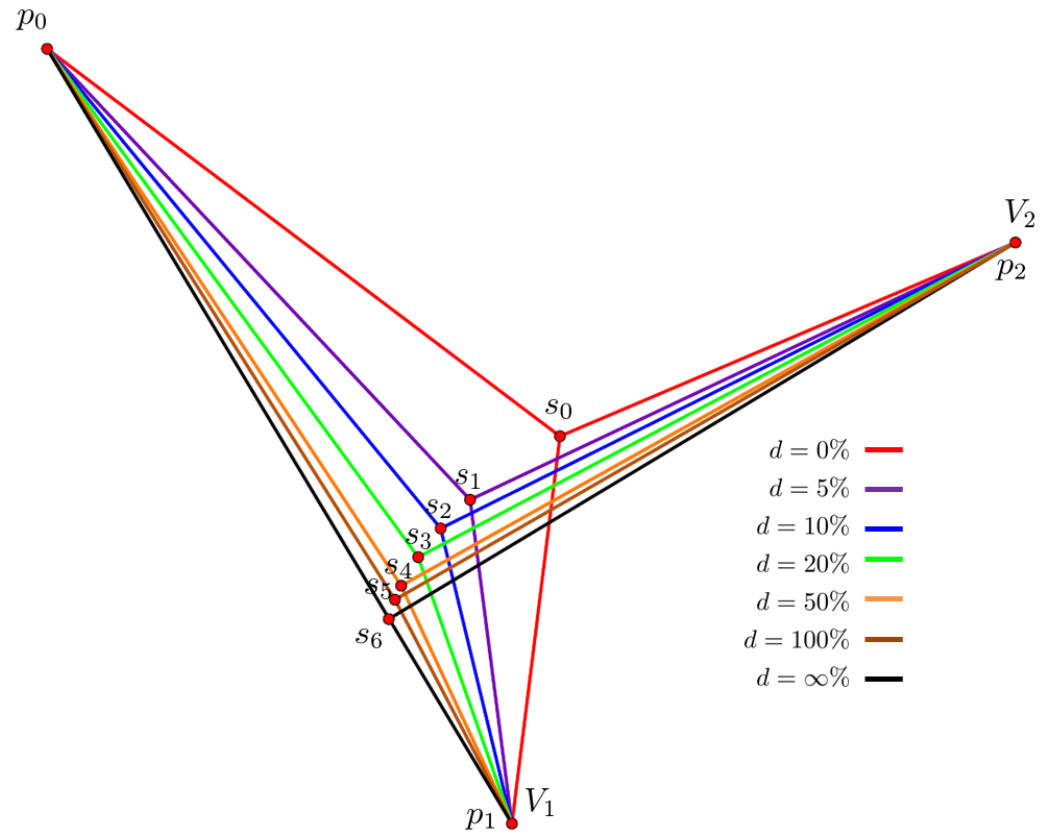

Fig. 7 The optimal locations of the discounted Steiner point for Case 2

Table 2 Variation of the NPV for a range of discount rates for Case 2

\begin{tabular}{cccccc}
\hline $\begin{array}{c}\text { Discount } \\
\text { rate } / \% \text { p.a. }\end{array}$ & $\begin{array}{c}\text { Location } \\
\text { of the DSP }\end{array}$ & $\begin{array}{c}\theta \\
/ \mathrm{deg}\end{array}$ & $\begin{array}{c}l_{2} \\
/ \mathrm{m}\end{array}$ & $\begin{array}{c}\text { NPV } \\
/ \$ \mathrm{M}\end{array}$ & $\begin{array}{c}\text { Improvement } \\
/ \$\end{array}$ \\
\hline 0 & $s_{0}=(662,0,500)$ & 120 & 638 & 93.120 & 0 \\
5 & $s_{1}=(546,0,418)$ & 108 & 777 & 90.221 & 159000 \\
10 & $s_{2}=(508,0,381)$ & 102 & 827 & 87.674 & 435000 \\
20 & $s_{3}=(479,0,344)$ & 98 & 870 & 83.183 & 995000 \\
50 & $s_{4}=(457,0,307)$ & 94 & 908 & 72.778 & 2269000 \\
100 & $s_{5}=(449,0,289)$ & 92 & 923 & 61.269 & 3449000 \\
$\infty$ & $s_{6}=(441,0,264)$ & 90 & 943 & 0 & 0 \\
\hline
\end{tabular}

The results are shown in Fig. 6 and Table 1 for Case 1 and in Fig. 7 and Table 2 for Case 2. In the tables, DSP refers to the Discounted Steiner Point. Fig. 6 and Fig. 7 illustrate the optimal trees for the discount rates that were considered. In Tables 1 and 2, for zero and infinite discount rates the optimal values of $\theta$ are $2 \pi / 3$ and $\pi / 2$, respectively, which verifies Lemmas 3 and 4 for this set of data. 
We have compared the NPV by placing the discounted Steiner point at the classical Steiner point for all the discount rates as shown in the last column (Improvement) of the Tables 1 and 2. For positive discount rates the discounted Steiner point gives better NPV, and the improvement in the NPV increases with increasing discount rate.

\section{Conclusion}

This paper proposes an efficient algorithm to locate the discounted Steiner point for a simple discounted Steiner tree. We prove the uniqueness of the discounted Steiner point and the convergence of the discounted Steiner point algorithm subject to realistic values of the input parameters. We show in a maximum NPV tree the paths from the discounted Steiner point to the first terminal and the second terminal make equal angles with the path from the discounted Steiner point to the third terminal (surface portal). For zero discount rate, the problem is reduced to a length minimization problem and the solution is indeed the classical Steiner point. When the DSPA is applied to typical underground mine data, it yields higher NPV compared with the placement of the Steiner point at the classical position. This algorithm can be applied in a real mine situation when the resource points and the surface portal, or breakout point from existing infrastructure, lie in a plane that is nearly horizontal and hence the gradient constraint would be satisfied.

In future, a new algorithm will be developed to locate a single discounted Steiner point with more realistic constraints such as the gradient constraint, which is the most important physical constraint on the access network, and the optimal configurations will be studied.

The discounted Steiner point algorithm will also be extended to locate multiple discounted Steiner points in a network. The idea is to use the discounted Steiner point algorithm iteratively. Initial numerical trials show that the new algorithm works satisfactorily and converges for two discounted Steiner points. A similar iterative process will be investigated to locate multiple Steiner points.

\section{References}

1. Hwang, F.K., Richards, D.S., Winter, P.: The Steiner Tree Problem. Elsevier Science Publishers (1992)

2. Brazil, M., Rubinstein, J.H., Thomas, D.A., Weng, J.F., Wormald, N.C.: Gradient constrained minimum networks (I). Fundamentals. Journal of Global Optimization 21(2), 139$155(2001)$

3. Brazil, M., Thomas, D.A.: Network optimisation for the design of underground mines. Networks 49(1), 40-50 (2007)

4. Lane, K.F.: The Economic Definition of Ore - Cutoff Grade in Theory and Practice. Mining Journal Books Limited, London (1988)

5. Nehring, M., Topal, E.: Production Schedule Optimisation in Underground Hard Rock Mining Using Mixed Integer Programming. Project Evaluation Conference, The Australasian Institute of Mining and Metallurgy 2007(4), 169-175 (2007)

6. Nehring, M., Topal, E., Little, J.: A new mathematical programming model for production schedule optimisation in underground mining operations. Journal of the South African Institute of Mining and Metallurgy 110(8), 437-447 (2010)

7. Newman, A.M., Kuchta, M.: Using aggregation to optimize long-term production planning at an underground mine. European Journal of Operational Research 176(2), 1205-1218 (2007) 
8. Newman, A.M., Rubio, E., Caro, R., Weintraub, A., Eurek, K.: A Review of Operations Research in Mine Planning. Interfaces 40(3), 222-245 (2010)

9. Montalant, F., et al.: Analyse des infiniment petits, pour lintelligence des lignes courbes. chez Francois Montalant (1716) 


\section{University Library}

\section{- M M I N E R VA A gateway to Melbourne's research publications}

Minerva Access is the Institutional Repository of The University of Melbourne

Author/s:

Sirinanda, KG;Brazil, M;Grossman, PA;Rubinstein, JH;Thomas, DA

Title:

Maximizing the net present value of a Steiner tree

Date:

2015

Citation:

Sirinanda, K. G., Brazil, M., Grossman, P. A., Rubinstein, J. H. \& Thomas, D. A. (2015).

Maximizing the net present value of a Steiner tree. Journal of Global Optimization, 62 (2), pp.391-407. https://doi.org/10.1007/s10898-014-0246-3.

Persistent Link:

http://hdl.handle.net/11343/282912 